\title{
H I bubbles surrounding southern optical ring nebulae: Anon (WR 23) and RCW 52
}

\author{
C. Cappa ${ }^{1,2, \star}$, V. S. Niemela ${ }^{2, \star \star}$, M. C. Martín ${ }^{1, *}$, and N. M. McClure-Griffiths ${ }^{3}$ \\ 1 Instituto Argentino de Radioastronomía, CC 5, 1894 Villa Elisa, Argentina \\ e-mail: ccappa@fcaglp.fcaglp.unlp.edu.ar \\ 2 Facultad de Ciencias Astronómicas y Geofísicas, Universidad Nacional de La Plata, Paseo del Bosque s/n, 1900 La Plata, \\ Argentina \\ 3 Australia Telescope National Facility, CSIRO; PO Box 76, Epping NSW 1710, Australia
}

Received 9 November 2004 / Accepted 17 January 2005

\begin{abstract}
We analyze the interstellar medium in the environs of two hot and massive stars, HD 92809 (=WR 23, WC6) and LSS 1887 (O8V), which ionize the optical ring nebulae Anon (WR 23) and RCW 52, respectively. Our analysis is based on neutral hydrogen (HI) $21 \mathrm{~cm}$ line data, which reveal interstellar bubbles surrounding the massive stars and their optical ring nebulae. The HI bubble related to WR 23 is $13.3 \mathrm{pc}$ in radius and is expanding at $10 \mathrm{~km} \mathrm{~s}^{-1}$. The associated atomic neutral mass is $830 M_{\odot}$. The HI structure related to LSS 1887 is about $6.3 \mathrm{pc}$ in radius, has an expansion velocity of $7 \mathrm{~km} \mathrm{~s}^{-1}$ and an associated atomic neutral mass of $100 M_{\odot}$. These HI features are the neutral counterparts of the optical ring nebulae and were mainly created by the action of the stellar winds of the massive stars on their environs. The dynamical age of the HI bubble around WR $23\left(7 \times 10^{5} \mathrm{yr}\right)$ suggests that it was created during the WR phase of stellar evolution. However, the large tangential motions of WR 23 and LSS 1887 suggest that part of the observed optical and HI structures may be due to a bow shock. The analysis of the distribution of emission in the far infrared and in the $\mathrm{CO}(1-0)$ molecular line in the environs of WR 23 and LSS 1887 reveals that there are also infrared and molecular counterparts of the detected HI bubbles.
\end{abstract}

Key words. ISM: bubbles - HII regions

\section{Introduction}

Along with supernova ejecta, the stellar winds from massive stars, mainly those of spectral types $\mathrm{O}$, Of and Wolf-Rayet (WR), transfer large amounts of mechanical energy and momentum into the ambient medium. Stellar winds sweep up and compress the interstellar material creating expanding shells. In particular, WR stars - although relatively few within our Galaxy (e.g. van der Hucht 2001) - contribute up to $70 \%$ of the momentum and $40 \%$ of the kinetic energy input from stellar winds within a radius of $3 \mathrm{kpc}$ from the Sun. This is followed closely by O-type stars, which contribute with about $25 \%$ of the kinetic energy (cf. Castor 1993).

Stellar winds from massive stars create interstellar bubbles. These structures have been modeled by several authors taking into account different interstellar environments and the mass loss history of the central star (e.g. Dyson 1989; García-Segura \& Mac Low 1995, and references therein). These bubbles can be detected in different spectral regimes: as optical ring shaped nebulae (e.g. Marston et al. 1994), as shell shaped thermal radio continuum sources (e.g. Goss \& Lozinskaya 1995), as neutral gas voids and expanding shells in the HI $21 \mathrm{~cm}$ line emission

\footnotetext{
$\star$ Member of Carrera del Investigador, CONICET, Argentina.

$\star \star$ Member of Carrera del Investigador, CIC-BA, Argentina.
}

distribution (e.g. Cappa et al. 2003) and as infrared shells (e.g. Mathis et al. 1992). Analysis of the atomic hydrogen distribution has allowed the identification of interstellar neutral gas bubbles surrounding about $20 \%$ of the Galactic WR stars and a large number of O-type stars. Thus, HI $21 \mathrm{~cm}$ line observations have proved to be a very useful tool to detect the compressed expanding outer layers of these interstellar bubbles (e.g. Cappa et al. 2003; Chu et al. 2004).

In this paper we analyze the interstellar environment of two massive stars in the giant HII region and OB stellar complex in Carina: the WR star HD 92809 (=WR 23 =LSS 1768) inside the optical filamentary nebula Anon (WR 23) (Lortet et al. 1980), and the O-type star LSS 1887 inside the HII region RCW 52 (Rodgers et al. 1960). In the present study, which is based on HI $21 \mathrm{~cm}$ line data and additional far infrared and radio continuum information, we report the detection of two new HI bubbles, namely those associated with Anon(WR 23) and RCW 52.

\section{The stars and their optical nebulae}

\subsection{WR23 and Anon (WR23)}

The WR star HD 92809 (=WR 23) is related to Car OB1 (cf. Lundström \& Stenholm 1984). Van der Hucht (2001) places 
the star at $3.2 \mathrm{kpc}$ according to distance determinations for early type stars belonging to $\operatorname{Tr} 14$ and $\operatorname{Tr} 16$ by Massey \& Johnson (1993). However, the distance to Car OB1 is still a matter of debate, with different authors deriving distances in the range from 1.8 to $3.2 \mathrm{kpc}$ (e.g. Feinstein et al. 1973; Walborn 1995).

WR 23 is a WC6 type star with galactic coordinates $(l, b)=$ (286 $466^{\prime} 8,-0^{\circ} 1$ '.8) (cf. van der Hucht 2001). Its optical ring nebula (Anon WR 23) was first identified by Lortet et al. (1980) as a filamentary shell of about $30^{\prime}$ in diameter, with the WR star projected close to the center of the nebula (Fig. 1, top panel). The morphology, nebular $\mathrm{H} \alpha$ kinematics and stellar content of this region have been discussed by Georgelin et al. (1986). The optical filamentary shell surrounding WR 23 is emitting in [SII] $\lambda \lambda 6716,6731$ nebular emission lines, as seen in the composite three-color image published by Smith et al. (2000), in their study of the large-scale structure of the Carina Nebula.

Proper motion values of WR 23 from the Tycho-2 catalog are $\mu_{\alpha} \cos \delta=-9.6 \pm 1.5 \operatorname{mas~yr}^{-1}, \mu_{\delta}=+2.2 \pm 1.5 \mathrm{mas} \mathrm{yr}^{-1}$ (Hog et al. 2000), indicating that WR 23 has a significant tangential motion, directed approximately toward the brightest part of the optical nebula as indicated by the arrow in the top panel of Fig. 1.

Radio continuum emission from the ionized gas in Anon(WR 23) was detected in the $4.9 \mathrm{GHz}$ survey obtained with an angular resolution of 4 '.1. by Haynes et al. (1979), as shown in Fig. 1b in Lortet et al. (1980).

The region displays quite complex kinematics. Based on interferometric $\mathrm{H} \alpha$ profiles, Chu (1982) and Georgelin et al. (1986) found that the ionized gas has negative velocities, generally in the range -31 to $-1 \mathrm{~km} \mathrm{~s}^{-1}$, with a mean velocity of about $-20 \mathrm{~km} \mathrm{~s}^{-1}$, indicating that the nebula is related to the Carina complex. (All velocities in this paper are referred to the LSR). The expansion velocity and the dynamical age of the $\mathrm{H} \alpha$ nebula around WR 23 were estimated as $15-30 \mathrm{~km} \mathrm{~s}^{-1}$ and (2-4) $\times 10^{5} \mathrm{yr}$, respectively (Georgelin et al. 1986; Chu 1982).

Marston (1996) analyzed IRAS images at 60 and $100 \mu \mathrm{m}$ (with angular resolutions of about $6^{\prime}$ and $4^{\prime}$, respectively) and noted an infrared shell coincident in position with the optical filamentary nebula Anon(WR 23).

\subsection{LSS 1887 and RCW 52}

LSS $1887\left(\mathrm{OB}^{+}, \mathrm{CPD}-57^{\circ} 3781 \mathrm{~S}\right)$ is an O-type star located at $(l, b)=\left(287^{\circ} 14^{\prime} .4,+0^{\circ} 21^{\prime}\right)$. The star was classified as O8 by Crampton (1972) and as O7V(n) by Vijapurkar \& Drilling (1993). LSS 1887 is the exciting star of the HII region RCW 52 (=Gum 32) of about $6^{\prime}$ in diameter. Georgelin et al. (1986) include the exciting star in Car OB1. The proper motion of LSS 1887 from the Tycho-2 catalog (Hog et al. 2000) is $\mu_{\alpha} \cos \delta=-0.7 \pm 2.8 \mathrm{mas} \mathrm{yr}^{-1}, \mu_{\delta}=+8.4 \pm 2.6 \mathrm{mas} \mathrm{yr}^{-1}$. The direction of its motion is shown in Fig. 1.

The optical appearance of RCW 52 is a partial ring (shown in detail in the bottom panel of Fig. 1), with the massive star, indicated by a cross, close to its center. RCW 52 was detected in radio continuum emission at $5 \mathrm{GHz}$ and in $\mathrm{H} 110 \alpha$ radio recombination lines with a radial velocity of $-18 \mathrm{~km} \mathrm{~s}^{-1}$
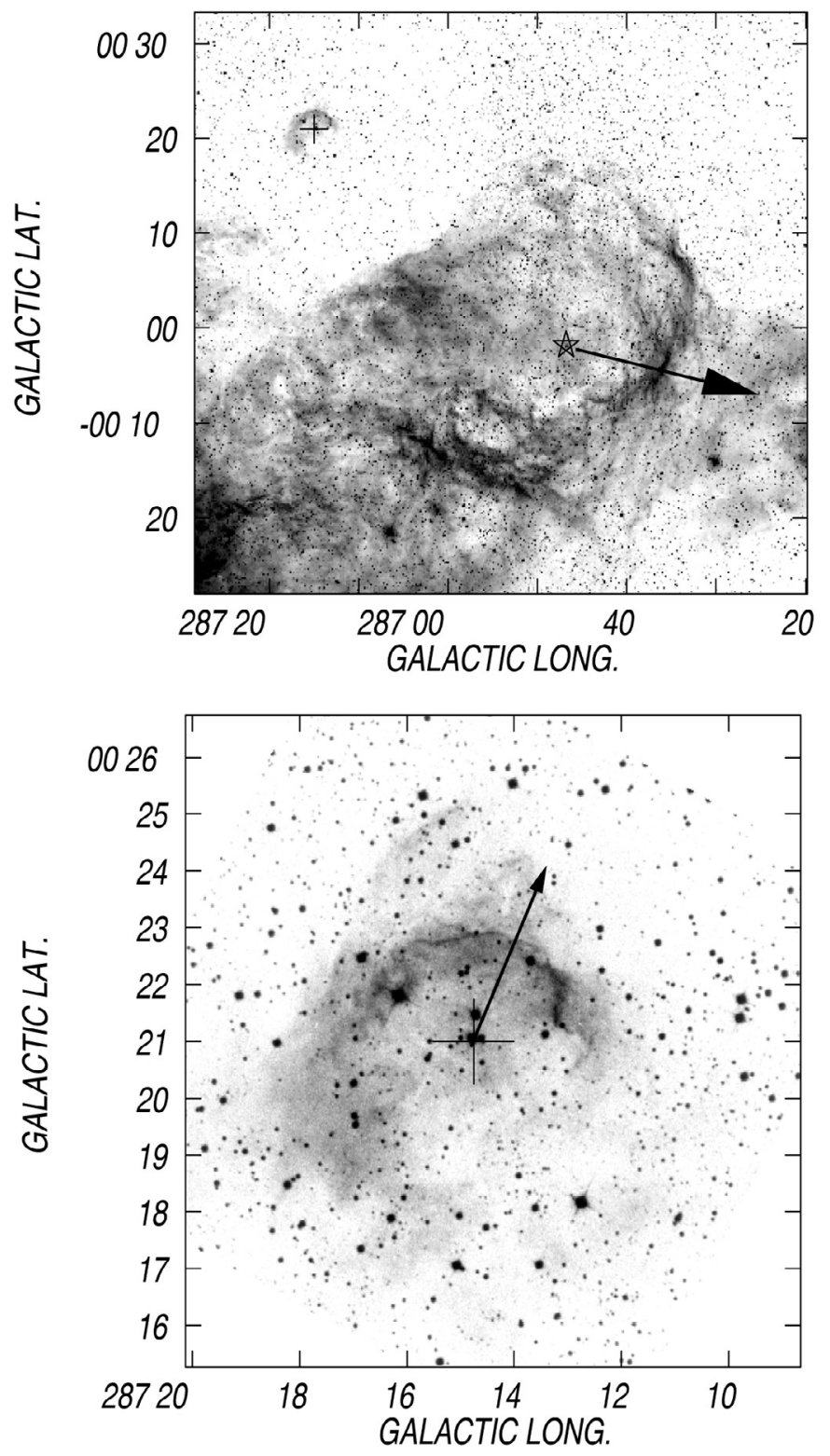

Fig. 1. Top panel: DSS $\mathrm{R}$ image showing the ring nebulae Anon (WR 23) and RCW 52 around the stars WR 23 and LSS 1887. The positions of WR 23 and LSS 1887 are indicated by the star symbol and the cross, respectively. The arrows show the direction of the proper motion of the stars. Bottom panel: full resolution DSS R image of RCW 52. The intensity units are arbitrary.

(Caswell \& Haynes 1987), in agreement with the $\mathrm{H} \alpha$ velocity listed by Georgelin et al. (1986, see their Table 3a).

\section{Data bases}

\subsection{Radio data}

We have analyzed the neutral gas distribution in the environs of the optical nebulae using HI $21 \mathrm{~cm}$ line emission in an area of $2^{\circ} \times 2^{\circ}$ centered approximately at the position of WR 23 . The HI data are part of the Southern Galactic Plane Survey (SGPS) and were obtained using the Australia Telescope Compact Array (ATCA) and the Parkes Radiotelescope (short spacing 
Table 1. Radio data: relevant parameters.

\begin{tabular}{lc}
\hline \hline HI data & \\
\hline$(l, b)$ Field center & $(286.8,+0.1)$ \\
Synthesized beam & $2 ! 4 \times 2.1$ \\
Number of channels & 256 \\
Velocity coverage & $(-190,+230) \mathrm{km} \mathrm{s}^{-1}$ \\
Velocity resolution & $1.64 \mathrm{~km} \mathrm{~s}^{-1}$ \\
RMS noise level & $1.6 \mathrm{~K}$ \\
\hline CO data & \\
\hline Angular resolution & 8.8 \\
Velocity coverage & $(-160,+160) \mathrm{km} \mathrm{s}^{-1}$ \\
Velocity resolution & $1.3 \mathrm{~km} \mathrm{~s}^{-1}$ \\
RMS noise level & $0.2 \mathrm{~K}$ \\
\hline
\end{tabular}

information). The HI data have a synthesized beam of $2 ! 4 \times 2 ! 1$, a velocity resolution of $0.82 \mathrm{~km} \mathrm{~s}^{-1}$ and an rms noise of $2.3 \mathrm{~K}$. A complete description of the HI data can be found in McClure-Griffiths (2001).

To improve the signal-to-noise ratio, the original data were averaged taking pairs of consecutive line images, resulting in a velocity resolution of $1.6 \mathrm{~km} \mathrm{~s}^{-1}$ and an rms noise of $1.6 \mathrm{~K}$. Table 1 displays the most important observational parameters of the final HI data cube.

We have also analyzed the distribution of the molecular gas based on the CO survey by Dame et al.(2001). The main observational parameters of this data base are listed in Table 1.

Radio continuum data available at $4.85 \mathrm{GHz}$ from the Parkes-MIT-NRAO (PMN) Southern Radio Survey and the Haynes et al. (1979) survey were also analyzed. The angular resolution of both surveys is $5^{\prime}$. Additional information on the PMN survey can be found in Condon et al. (1993).

\subsection{Infrared data}

We have also investigated the dust distribution in the region with high-resolution (HIRES) data obtained through IPAC ${ }^{1}$. The IR data in the IRAS bands at 60 and $100 \mu \mathrm{m}$ have angular resolutions of 1.5 and 1.'8.

\section{Neutral gas emission distribution in the line of sight to Anon (WR 23) and RCW 52}

Within the selected region, significant $\mathrm{HI}$ emission was detected from -50 to about $+110 \mathrm{~km} \mathrm{~s}^{-1}$. Circular galactic rotation models (e.g. Brand \& Blitz 1993) predict negative radial velocities in the range -11 to $0 \mathrm{~km} \mathrm{~s}^{-1}$ for distances closer than 5-6 kpc in this section of the Galaxy. However, HII regions and molecular clouds display negative velocities of up to $-40 \mathrm{~km} \mathrm{~s}^{-1}$ (see the observed velocity pattern displayed in Fig. 2b of Brand \& Blitz 1993). Thus, in order to investigate the HI gas linked to WR 23 and LSS 1887 we will concentrate on the distribution of the HI emission at $v \geq-50 \mathrm{~km} \mathrm{~s}^{-1}$.

The analysis of the neutral gas distribution in the environs of the nebulae showed the presence of cavities in the

\footnotetext{
${ }^{1}$ IPAC is funded by NASA as part of the IRAS extended mission under contract to Jet Propulsion Laboratory (JPL) and California Institute of Technology (Caltech).
}
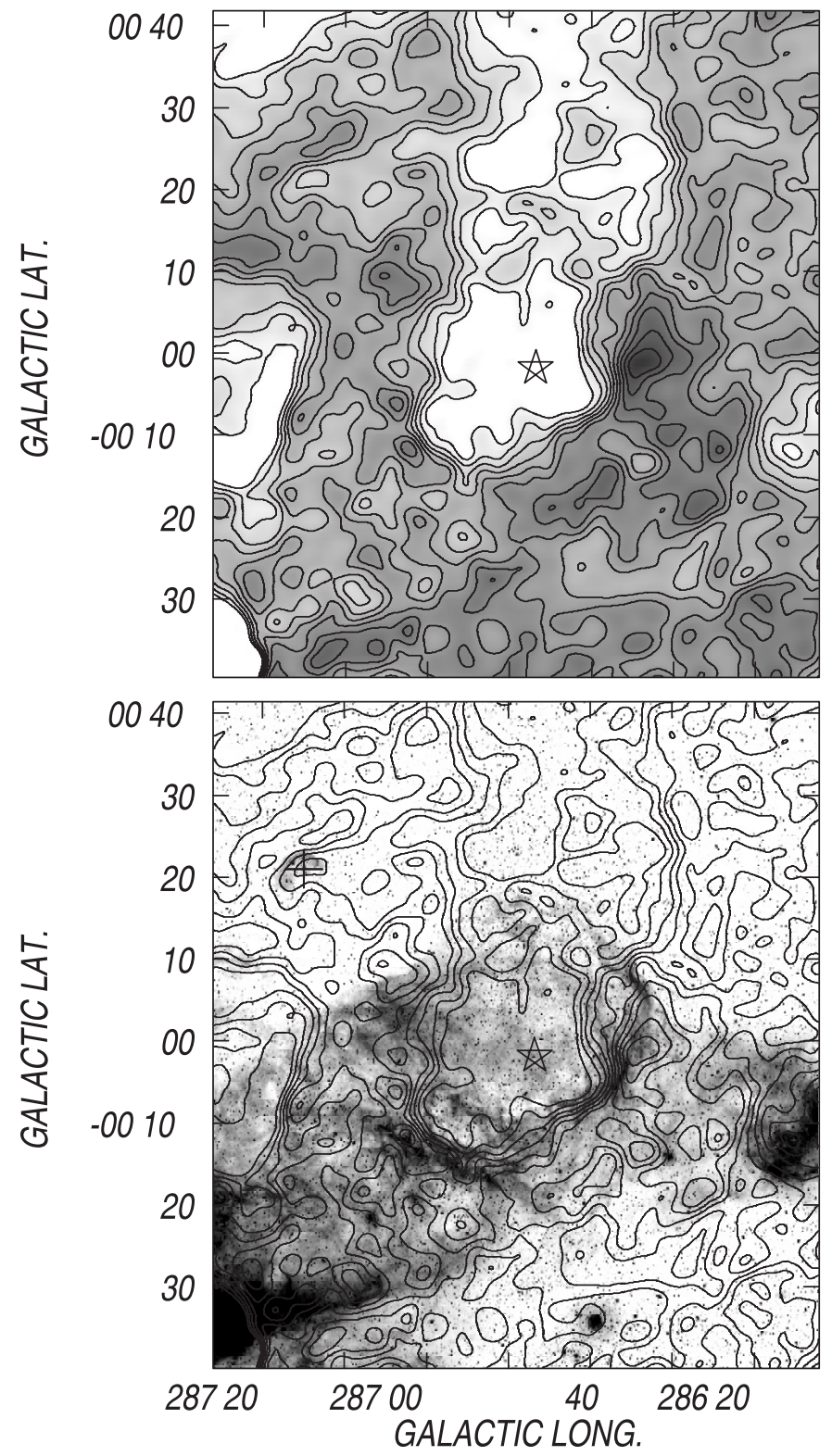

Fig. 2. Top: HI column density distribution showing the HI bubble associated with Anon(WR 23) within the velocity range -21.4 to $-12.0 \mathrm{~km} \mathrm{~s}^{-1}$. The grayscale is from $12.0 \times 10^{20}$ to $23.0 \times 10^{20} \mathrm{~cm}^{-2}$. The contour lines are from $12.4 \times 10^{20}$ to $18.0 \times 10^{20} \mathrm{~cm}^{-2}$ in steps of $0.8 \times 10^{20} \mathrm{~cm}^{-2}$. As in Fig. 1, the star indicates the position of WR 23 . Bottom: overlay of the DSS R (grayscale) and HI (contours) showing a close correspondence of neutral and ionized gas emissions.

HI distribution at about $v \sim-17 \mathrm{~km} \mathrm{~s}^{-1}$ towards both WR 23 and LSS 1887.

In the following sections we will analyze both Hi structures separately, along with their counterparts at other frequencies and their connection to the optical nebulae.

\section{The interstellar bubble associated with WR 23}

\subsection{The neutral gas}

A close inspection of the individual HI images shows that WR 23 appears projected onto a minimum in the HI emission 
distribution detected within the velocity range -25 to $-12 \mathrm{~km} \mathrm{~s}^{-1}$. The cavity is surrounded by patchy regions of enhanced neutral emission which define an envelope. The cavity and the surrounding shell are clearly identified in the top panel of Fig. 2, which shows the HI column density distribution within the velocity interval -22.0 to $-12.0 \mathrm{~km} \mathrm{~s}^{-1}$, where the structure is best defined. WR 23 appears projected close to the center of the HI hole. This structure, centered approximately at $\left(286^{\circ} 50^{\prime},-0^{\circ} 3^{\prime}\right)$, can be followed at lower negative velocities up to $-29 \mathrm{~km} \mathrm{~s}^{-1}$.

The bottom panel of Fig. 2 displays an overlay of the HI and the optical line emissions. The comparison of the DSS R and HI images shows an excellent correspondence between the optical filaments and the inner border of the HI cavity from $\left(286^{\circ} 35^{\prime},+0^{\circ} 8^{\prime}\right)$ to $\left(287^{\circ} 0^{\prime},-0^{\circ} 10^{\prime}\right)$.

Towards higher galactic latitudes, where the optical ring nebula Anon (WR 23) appears incomplete, the HI emission is also weaker. The weak enhancement in HI emission in this section of the neutral envelope suggests that the HI cavity is connected to the hole centered at $\left(286^{\circ} 40^{\prime},+0^{\circ} 20^{\prime}\right)$. The presence of weak optical filaments in the high galactic latitude region of the nebula gives additional support to this interpretation.

The HI structure has a systemic velocity, defined as the velocity where the cavity and the surrounding envelope present their largest dimension, of about $-17 \mathrm{~km} \mathrm{~s}^{-1}$, similar to the mean $\mathrm{H} \alpha$ velocity, namely $-20 \mathrm{~km} \mathrm{~s}^{-1}$, found by Georgelin et al. (1986) and the velocity of radio recombination lines (-18 $\mathrm{km} \mathrm{s}^{-1}$, Caswell \& Haynes 1987).

The clear morphological correspondence between the optical nebula Anon (WR 23) and the HI structure, as well as the similar velocities found for the neutral and the ionized gas features, indicate that the HI shell is the atomic gas counterpart of the optical nebula.

\subsection{Counterparts of the HI bubble around WR23 at other wavelengths}

The ionized gas in Anon (WR 23) is also seen in radio continuum emission at $4.85 \mathrm{GHz}$ both in the PMN survey and in the Haynes et al. (1979) survey. The region with thin and bright ionized optical filaments seen in Fig. 1 at $l \leq 286^{\circ} 50^{\prime}$ have a clear radio continuum counterpart, as well as some faint optical filaments near $\left(286^{\circ} 40^{\prime},+0^{\circ} 8^{\prime}\right)$ (see Fig. $1 \mathrm{~b}$ in Lortet et al. 1980).

The distribution of the interstellar dust is revealed by the emission in the far infrared. The top panel of Fig. 3 displays the HIRES IR emission at $100 \mu \mathrm{m}$, while the bottom panel shows an overlay of the IR and optical images. The figure shows that the interstellar bubble is also detected as a shell in the far IR, with the IR contours closely following the bright optical filaments. A comparison of Figs. 2 and 3 shows that there is also a significant correlation between the HI and IR distributions, with the HI and IR shells broken near $b \simeq+0^{\circ} 10^{\prime}$. The far IR emission distribution at $60 \mu \mathrm{m}$, not shown here, is similar to the distribution at $100 \mu \mathrm{m}$.

The distribution of the molecular material in the environs of the stars and their optical nebulae was investigated by

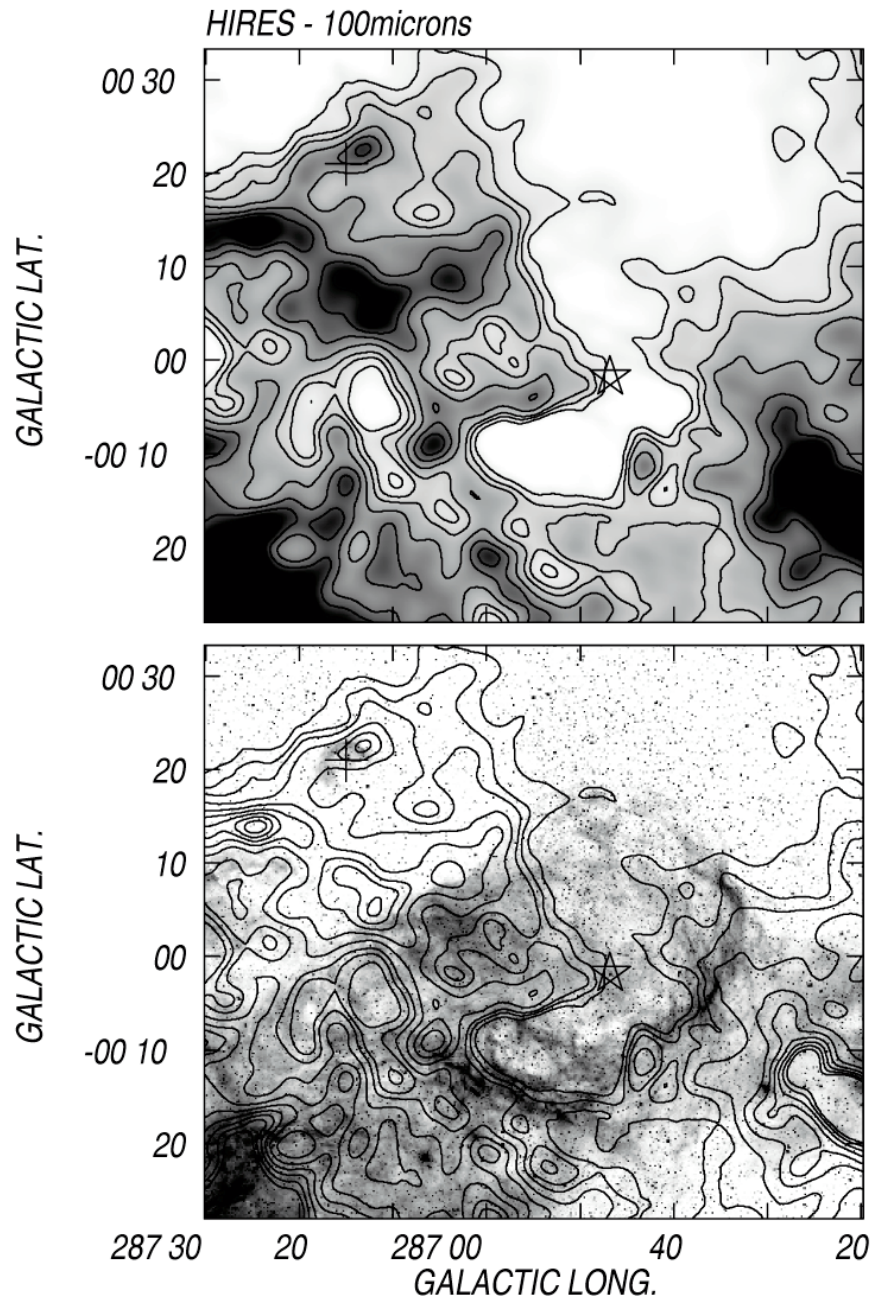

Fig. 3. Top panel: far infrared IRAS HIRES emission distribution at $100 \mu \mathrm{m}$ in the region around WR 23 and RCW 52. The grayscale corresponds to $0.002 \times 10^{3}$ to $1.3 \times 10^{3} \mathrm{MJy} \mathrm{ster}^{-1}$. The contour lines are $0.1,0.2,0.3,0.5,0.75,1.00,1.25,1.50,1.75$ and $2.00 \mathrm{MJy} \mathrm{ster}^{-1}$. The star and the cross mark the positions of WR 23 and LSS 1887, respectively. Bottom panel: overlay of the far IR contour lines of the upper panel and the optical emission as seen in Fig. 1 (top panel).

analyzing the $\mathrm{CO}(1-0)$ line emission from the survey by Dame et al. (2001). Figure 4 (top panel) displays the distribution of the molecular gas emission within the velocity interval -24.1 to $-5.9 \mathrm{~km} \mathrm{~s}^{-1}$. The bottom panel shows an overlay of the $\mathrm{CO}$ (contours) and the HI (grayscale) images. Figure 4 reveals a region lacking molecular material almost coincident with both the HI voids linked to WR 23 and the one centered at higher galactic latitudes $\left(286^{\circ} 40^{\prime},+0^{\circ} 20^{\prime}\right)$. The molecular cavity is also observed in the maps by Grabelsky et al. (1988).

The agreement both in velocity between the HI and CO shells and in position among the HI, CO and IR shells indicate that the voids and shells observed in the distributions of the neutral components (HI, CO and dust) are the neutral gas counterparts of the optical ring nebula and have the same origin, i.e., are the result of the action of the stellar winds and ionizing radiation on the interstellar material. 

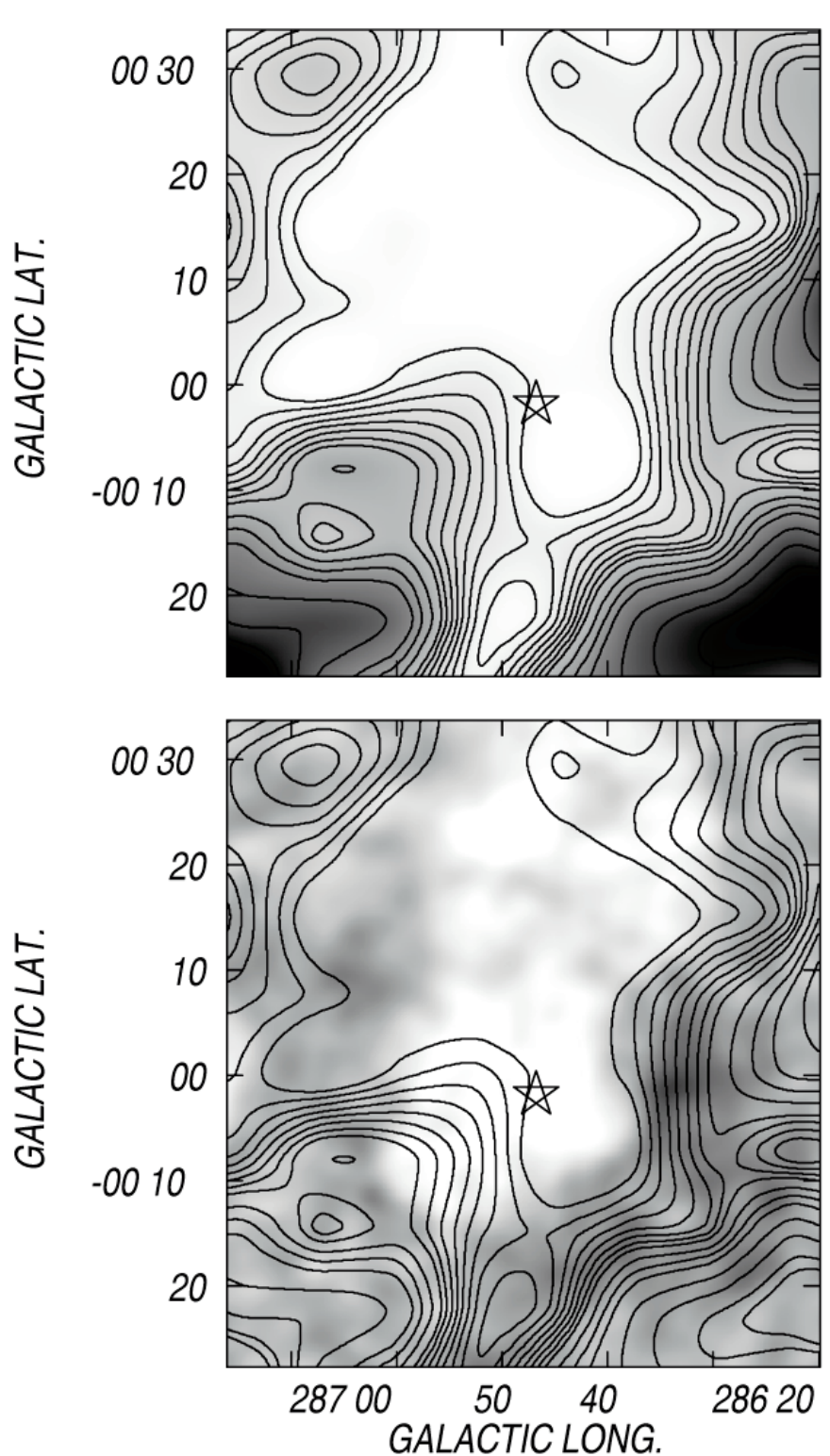

Fig. 4. Top: mean $\mathrm{CO}$ brightness temperature in the environs of Anon (WR 23) within the velocity range -24.1 to $-5.9 \mathrm{~km} \mathrm{~s}^{-1}$. The grayscale corresponds to 2.0 to $32.0 \mathrm{~K}$. The contour lines are from 3.0 to $9.0 \mathrm{~K}$ in steps of $1.0 \mathrm{~K}, 12.0$ to $20.0 \mathrm{~K}$ in steps of $2.0 \mathrm{~K}$, and 24.0 to $32.0 \mathrm{~K}$ in steps of $4.0 \mathrm{~K}$. Bottom: overlay of the $\mathrm{CO}$ (contours) and $\mathrm{HI}$ (grayscale) emissions.

\subsection{Interaction of ionized and neutral gas}

As seen in Figs. 2 and 4, the presence of neutral atomic and molecular material close to the sharp emission borders of the optical nebula suggests that the ionized gas is interacting with the neutral material. This is particularly noticeable close to $286^{\circ} 35^{\prime},-0^{\circ} 5^{\prime}$, where a strong brightness temperature gradient in the HI emission distribution is present. Here, the borders of both the $\mathrm{CO}$ and $\mathrm{HI}$ material coincide with the brightest optical region.

The components of the stellar proper motion of WR 23 in the galactic coordinates system are $\mu_{l} \cos b=-9.5 \pm$ $1.5 \mathrm{mas} \mathrm{yr}^{-1}$ and $\mu_{b}=-2.6 \pm 1.5 \mathrm{mas} \mathrm{yr}^{-1}$. The direction of the tangential motion, which is indicated in Fig. 1, approximately points towards the brightest optical region in the nebula. Values referred to LSR are $\left(\mu_{l} \cos b\right)_{\mathrm{LSR}}=-8.3 \pm$ $1.5 \mathrm{mas} \mathrm{yr}^{-1}$ and $\left(\mu_{b}\right)_{\mathrm{LSR}}=-0.9 \pm 1.5 \mathrm{mas} \mathrm{yr}^{-1}$. Adopting a distance $d=2.5 \mathrm{kpc}$ for WR 23, the components of the tangential velocity referred to LSR turn out to be $v_{l}=-98 \pm 20 \mathrm{~km} \mathrm{~s}^{-1}$ and $v_{b}=-11 \pm 18 \mathrm{~km} \mathrm{~s}^{-1}$, with the quoted errors due to uncertainties in the proper motions and in the distance. The large tangential motion of WR 23 supports the idea that at least part of the observed optical and HI structures might be due to a bow shock originated by the stellar motion.

\section{The $\mathrm{HI}$ bubble related to RCW 52}

\subsection{The neutral gas}

The neutral gas distribution in the environs of LSS 1887 as observed in HI $21 \mathrm{~cm}$ line data shows a small region lacking neutral hydrogen within the velocity interval -22 to $-12 \mathrm{~km} \mathrm{~s}^{-1}$ encircled by an almost complete envelope. The void and the shell can be identified in the top panel of Fig. 5, which displays the HI column density distribution within the velocity range -21.4 to $-12.0 \mathrm{~km} \mathrm{~s}^{-1}$. The central panel of Fig. 5, which shows an overlay of optical and HI images, reveals a clear correspondence between the optical nebular arc (i.e. RCW 52) and the inner border of the HI envelope. LSS 1887 is located about $15^{\prime}$ from the center of the neutral gas structure, close to the border of the region with highest HI density gradient, as is the case for many other central stars of Hi bubbles (cf. Cappa \& Herbstmeier 2000). The position of the optical nebula close to the borders of the HI void is also typical in wind blown bubbles.

\subsection{Counterparts of the HI bubble around RCW52 at other wavelengths}

The bottom panel of Fig. 5 displays an overlay of the $\mathrm{CO}$ emission distribution within the velocity range -24.1 to $-16.3 \mathrm{~km} \mathrm{~s}^{-1}$ (grayscale) and the HI contour lines. CO emission at these velocities close to RCW 52 was first detected by Grabelsky (1985). This image suggests the existence of a molecular counterpart of the atomic shell. Although the $\mathrm{CO}$ data were obtained with poorer angular resolution than the HI data, the COclouds broadly appear to follow the higher galactic longitude border of the neutral atomic shell surrounding RCW 52.

As pointed out in Sect. 2.2, RCW 52 is detected in the radio continuum emission at $4.85 \mathrm{GHz}$, both in the PMN and in the Haynes et al. (1979) surveys, where the radio source appears rather isolated from the background emission. The lack of a shell structure in the radio continuum data could be explained taking into account the poorer angular resolution of the data in comparison with the size of RCW 52 (see Fig. 1).

The IRAS HIRES IR emission at $100 \mu \mathrm{m}$ shows the presence of a void and a shell in the interstellar dust distribution around LSS 1887 (see Fig. 3). Two different components contribute to the emission: the strong IR emission at $(l, b)=\left(287^{\circ} 15^{\prime}\right.$, $\left.+0^{\circ} 22^{\prime}\right)$ correlates with the ionized region, while the more extended IR emission regions in a shell-shaped configuration correlate with the HI shell surrounding RCW 52. 

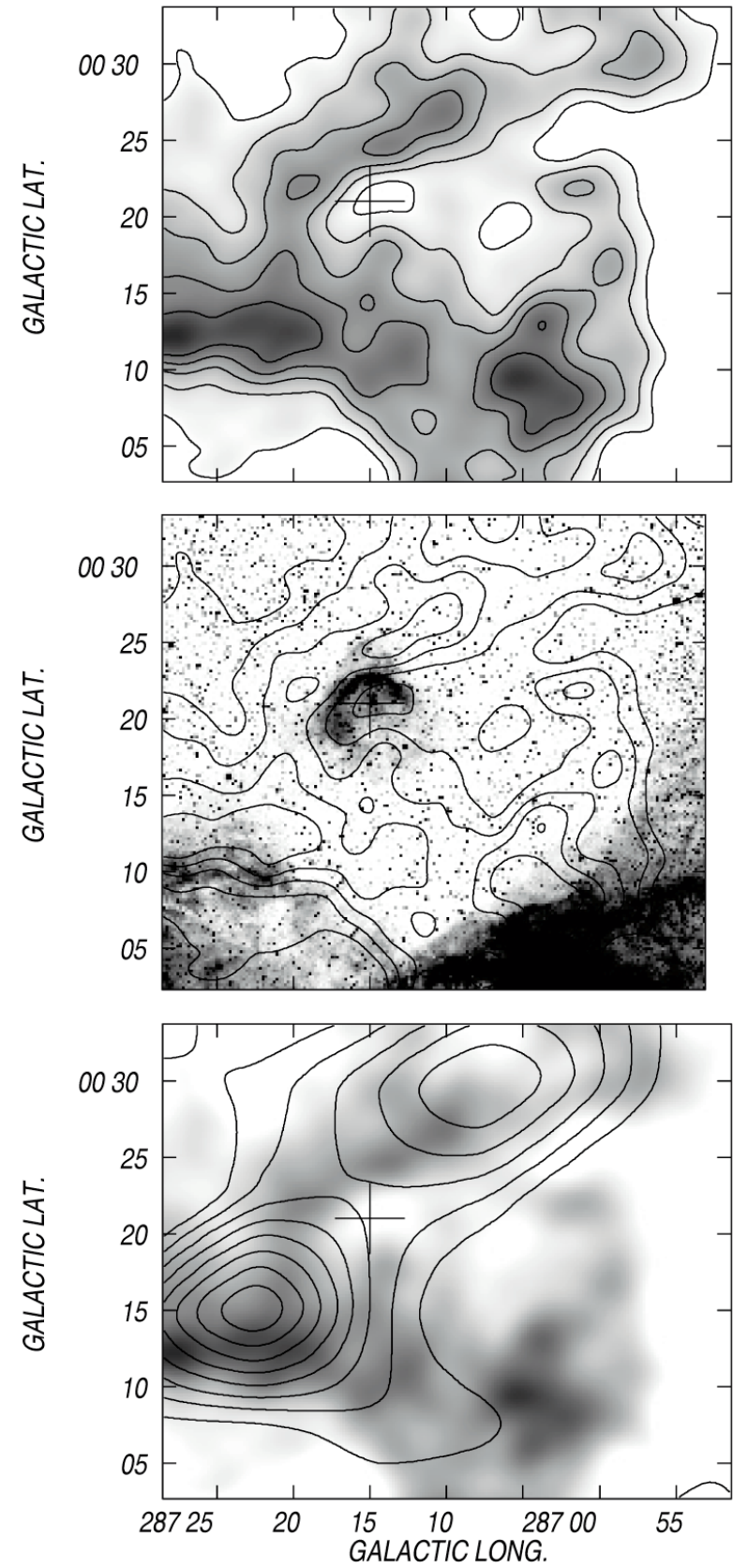

Fig. 5. Top: HI column density distribution in the environs of the optical ring nebula RCW 52 surrounding LSS 1887 within the velocity range -21.4 to $-12.0 \mathrm{~km} \mathrm{~s}^{-1}$. The grayscale corresponds to $14.0 \times 10^{20}$ to $19.0 \times 10^{20} \mathrm{~cm}^{-2}$. The contour lines are from $14.0 \times 10^{20}$ to $17.2 \times 10^{20} \mathrm{~cm}^{-2}$ in steps of $0.8 \times 10^{20} \mathrm{~cm}^{-2}$. The cross indicates the position of LSS 1887. Central: overlay of the optical image of the top panel of Fig. 1 (grayscale) and the HI (contours) emission. Bottom: overlay of the $\mathrm{HI}$ (grayscale) and $\mathrm{CO}$ (contours) emissions. Contour lines correspond to mean $\mathrm{CO}$ brightness temperature within the velocity interval -24.1 to $-16.3 \mathrm{~km} \mathrm{~s}^{-1}$. The values of the contours are 3.0 to $10.0 \mathrm{~K}$ in steps of $1.0 \mathrm{~K}$.

\subsection{Interaction of ionized and neutral gas}

We have also estimated the tangential velocity of LSS 1887. The components of the proper motion are $\mu_{l} \cos b=-4.5 \pm$ 2.8 mas yr $^{-1}$ and $\mu_{b}=+7.1 \pm 2.6$ mas yr$^{-1}$. Here also, the tangential motion points towards the brightest region of the nebula, as is the case for WR 23. Adopting a distance $d=$ $2.5 \mathrm{kpc}$ for LSS 1887, the components of the tangential velocity referred to LSR amount to $v_{l}=-38 \pm 33 \mathrm{~km} \mathrm{~s}^{-1}$ and $v_{b}=-102 \pm 33 \mathrm{~km} \mathrm{~s}^{-1}$, showing that the star has a significant motion. Therefore, also RCW 52 may at least partly be a bow shock originated by the stellar motion.

The optical appearance of the HII region, filamentary and limb brightened, resembles some of the classical ring nebulae (like NGC 6888), suggesting the action of the stellar winds sweeping up the surrounding material. The presence of voids and shells in the distribution of both the neutral material and the dust indicates that this HII region is a ring nebula created by both ionization and mass flow of the O-type star LSS 1887 .

As in the case of NGC 2359 (Cappa et al. 1999), the distribution of the different gas components supports the idea of the HI gas being placed at the interface between the ionized and the molecular gas. Then, the neutral atomic material seems to have originated in the dissociation of the molecular gas. The distribution of the different gas components suggests that a photodissociation region (PDR) has developed at the surface of the interacting molecular cloud.

\section{Discussion}

\subsection{Main physical parameters of the bubbles}

The main physical parameters of the ionized and neutral gas and the interstellar dust associated with the bubbles are summarized in Table 2. In this table, the " $(l, b)$ center" indicates the centroid of the neutral atomic structures. The "velocity interval" corresponds to the range where the HI structures can be identified, with $v_{1}$ and $v_{2}$ being the lowest and highest velocities at which the features are detected. The "systemic velocity" indicates the velocity where the HI bubbles present their largest dimensions.

The quoted "expansion velocities" in Table 2 are lower limits. They were estimated as $v_{\exp }=\left(v_{2}-v_{1}\right) / 2+1.6 \mathrm{~km} \mathrm{~s}^{-1}$. The extra $1.6 \mathrm{~km} \mathrm{~s}^{-1}$ allows for the presence of $\mathrm{HI}$ in the caps of the expanding shells. These caps are not detected in the present case, probably because of their weak brightness temperature.

The presence of strong non-circular motions detected in the Carina spiral arm precludes deriving reliable kinematical distances. Consequently, the distance adopted for the HI structures is based on spectrophotometric information only. Taking into account published distances for Car OB1, we adopted $d=$ $2.5 \pm 0.5 \mathrm{kpc}$ for both structures (see Sect. 2).

The radius of each $\mathrm{HI}$ void was estimated from Figs. 2 and 5 following approximately the contour lines of $13.3 \times 10^{20}$ and $15.6 \times 10^{20} \mathrm{~cm}^{-2}$ (which correspond to the first open contours of the voids) for the structures related to WR 23 and LSS 1887, respectively. The radius of each HI bubble was evaluated from the position of the maxima in the neutral gas shells.

The neutral mass in the bubbles was obtained as mean values between the mass deficiencies in the voids and the mass excess in the shells, assuming the optically thin case and a typical interstellar He abundance of $10 \%$.

For the case of wind blown bubbles, the dynamical age can be estimated as $t_{\mathrm{d}}=0.56 \times 10^{6} R / v_{\exp }$ yr (McCray 1983), 
Table 2. Main parameters of the ionized and neutral bubbles associated with WR 23 and LS 1887.

\begin{tabular}{|c|c|c|}
\hline & Anon (WR 23) & RCW 52 \\
\hline \multicolumn{3}{|l|}{ Neutral atomic gas } \\
\hline$(l, b)$ center & $286^{\circ} 50^{\prime},-0^{\circ} 3^{\prime}$ & $287^{\circ} 8^{\prime},+0^{\circ} 20^{\prime}$ \\
\hline Velocity interval $v_{1}, v_{2}\left(\mathrm{~km} \mathrm{~s}^{-1}\right)$ & $-29,-12$ & $-22,-12$ \\
\hline HI systemic velocity $\left(\mathrm{km} \mathrm{s}^{-1}\right)$ & -17 & -17 \\
\hline Expansion velocity $v_{\exp }\left(\mathrm{km} \mathrm{s}^{-1}\right)$ & 10 & 7 \\
\hline Radius of the HI cavity (arcmin) & 11.1 & 5.5 \\
\hline Radius of the HI bubble (arcmin) & 18.3 & 8.6 \\
\hline Adopted distance $(\mathrm{kpc})$ & $2.5 \pm 0.5$ & $2.5 \pm 0.5$ \\
\hline Radius of the HI bubble $R$ (pc) & 13.3 & 6.3 \\
\hline Swept-up atomic mass $\left(M_{\odot}\right)$ & 830 & 70 \\
\hline Dynamical age $\left(10^{5} \mathrm{yr}\right)$ & 7 & 5 \\
\hline \multicolumn{3}{|l|}{ Ionized gas } \\
\hline Flux density (Jy) & 5.5 & 1.8 \\
\hline Angular radius (arcmin) & 13 & 3.5 \\
\hline Linear radius $(\mathrm{pc})$ & 9.5 & 2.5 \\
\hline Emission measure $\left(\mathrm{pc} \mathrm{cm}^{-6}\right)$ & 990 & 4500 \\
\hline Filling factor $f$ & $0.09-0.16$ & $0.15-0.20$ \\
\hline Ionized mass $\left(M_{\odot}\right)$ & $500-680$ & $65-75$ \\
\hline Electron density $\left(\mathrm{cm}^{-3}\right)$ & $15-20$ & $55-65$ \\
\hline \multicolumn{3}{|l|}{ Associated dust } \\
\hline Flux density at $60 \mu \mathrm{m}(\mathrm{Jy})$ & 4800 & 5000 \\
\hline Flux density at $100 \mu \mathrm{m}(\mathrm{Jy})$ & 7700 & 7100 \\
\hline Dust mass $\left(M_{\odot}\right)$ & 3 & 2 \\
\hline Color temperature $(\mathrm{K})$ & 35 & 36 \\
\hline Ambient density $\left(\mathrm{cm}^{-3}\right)$ & 2 & 2 \\
\hline \multicolumn{3}{|l|}{ Energetic budget } \\
\hline Stellar wind mechanical energy $E_{\mathrm{W}}\left(10^{49} \mathrm{erg}\right)$ & 46 & 2 \\
\hline Kinetic energy of the bubble $E_{\mathrm{k}}\left(10^{47} \mathrm{erg}\right)$ & 14 & 0.7 \\
\hline$\epsilon$ & 0.003 & 0.004 \\
\hline
\end{tabular}

where $R$ is the radius of the bubble (pc), $v_{\exp }$ is the expansion velocity $\left(\mathrm{km} \mathrm{s}^{-1}\right)$ and the coefficient is the deceleration parameter. The constant is a mean value between the energy and the momentum conserving cases.

For the ionized bubbles, the emission measure $E M$, the ionized mass $M_{\mathrm{i}}$ and the electron density $n_{\mathrm{e}}$ were obtained using the classical expressions by Mezger \& Henderson (1967) based on flux densities derived from the PMN survey for the case of Anon (WR 23) and from the Haynes et al. (1979) survey for RCW 52 because this region lacks PMN data. We assumed an electron temperature $T_{\mathrm{e}}=10^{4} \mathrm{~K}$. Radii and adopted filling factors $f$ of the ionized regions are listed in Table 2. $f$-values were derived assuming that Anon(WR 23) and RCW 52 are shells of $1.5-3^{\prime}$ and $0.7-1 \prime^{\prime} 4$ in thickness, respectively, and that the plasma occupies $\sim 30 \%$ of the surface of the shells. The derived ionized masses were multiplied by 1.27 to take into account the presence of singly ionized He (Goss \& Lozinskaya 1995).

The large amount of neutral and ionized mass indicates that most of the material in the envelope is swept-up interstellar matter.

The HIRES infrared images allow a rough estimate of the amount of interstellar dust $M_{\mathrm{d}}$ linked to the bubbles and their colour temperatures $T_{\mathrm{d}}$. The dust mass was derived from the
$100 \mu \mathrm{m}$ image using the derived flux densities at this wavelength and standard dust grain parameters (e.g. Draine \& Lee 1984). Dust temperatures correspond to $n=1.5-2$. This parameter $(n)$ is related to the dust absorption efficiency $\kappa_{v} \propto v^{n}$, normalized to $40 \mathrm{~cm}^{2} \mathrm{~g}^{-1}$ at $100 \mu \mathrm{m}$.

Finally, the ambient density $n_{0}$ was derived by uniformly distributing the neutral and ionized masses over the volume of the neutral gas bubbles.

The major source of error in radii and masses is the distance uncertainty. Uncertainties of $20 \%$ in the distance implies a $20 \%$ error in radius and $40 \%$ error in the derived masses. An error of $30 \%$ was adopted for the expansion velocity. Uncertainty in the derived ambient density is about $100 \%$.

The radii and expansion velocities of the HI bubbles around WR 23 and LSS 1887 are similar to those derived for other neutral gas bubbles around WR and O-type stars (see Table 2 in Cappa et al. 2003). The dynamical age derived for the HI bubble around WR 23 agrees with previous results by Chu (1982) and shows that the interstellar bubble has been blown during the present WR phase. Errors in dynamical ages are about $70 \%$. We believe that the strong wind from the WR phase of the star has swept up the material shed by the star during previous evolutionary stellar phases. 


\subsection{Energy budget}

To analyze whether WR 23 and LSS 1887 can provide the energy to form the interstellar bubbles, we estimated the mechanical energy $E_{\mathrm{w}}$ released into the ISM during the dynamical age $t_{\mathrm{d}}$ of the structures and compared it to the kinetic energy $E_{\mathrm{k}}$ of the structures.

The stellar wind mechanical energy $E_{\mathrm{w}}\left(=L_{\mathrm{w}} t_{\mathrm{d}}=\dot{M} V_{\mathrm{w}}^{2} t_{\mathrm{d}} / 2\right)$ released by WR 23 was estimated assuming a mass loss rate $\dot{M} \approx 1.3 \times 10^{-5} M_{\odot} \mathrm{yr}^{-1}$ (Nugis \& Lamers 2000) and a terminal wind velocity $V_{\mathrm{w}}=2250 \mathrm{~km} \mathrm{~s}^{-1}$ (Eenens \& Williams 1994) and the dynamical age from Table 2 . For LSS 1887 we adopted $\dot{M}=2 \times 10^{-6} M_{\odot} \mathrm{yr}^{-1}$ and $V_{\mathrm{w}}=1500 \mathrm{~km} \mathrm{~s}^{-1}$ approximately corresponding to its spectral type (Prinja et al. 1990; Lamers \& Leitherer 1993).

The kinetic energy of the interstellar bubbles $E_{\mathrm{k}}=$ $M_{\mathrm{b}} v_{\exp }^{2} / 2$ was derived from the expansion velocities listed in Table 2 and the total mass in the bubble $M_{\mathrm{b}}$, obtained as the sum of the atomic and ionized masses.

The ratio $\epsilon$ between the kinetic energy $E_{\mathrm{k}}$ and the mechanical energy $E_{\mathrm{w}}$ provided by the massive star are also listed in Table 2. Although the uncertainty in this value is large (at least $70 \%$ adopting a $30 \%$ error in the distance), it is clear that both massive stars are capable of blowing their own interstellar bubbles. Stellar wind energy may drain through the section of the HI shell near $b=+0^{\circ} 10^{\prime}$, thus reenergizing the cavity centered at $286^{\circ} 40^{\prime},+0^{\circ} 20^{\prime}$, and decreasing the available stellar wind energy.

In estimating the stellar wind luminosity, we have considered the contribution of WR 23 and LSS1887 only. Indeed, the search for other massive stars projected onto the HI cavities and shells that might have played a role in shaping the structures resulted in negative results. However, we note that LSS 1887 may be a multiple system as suggested by the image in Fig. 1.

For Anon(WR 23), a number of OB stars are seen in projection onto the HI shell: HD 92714 (=LSS 1753 at $286^{\circ} 37 ! 2$, +0 $0^{\circ} 6^{\prime} 6$ ), HD 303179 (=LSS 1737 at 286 $36^{\prime} \cdot 6$, $\left.-0^{\circ} 5.4\right)$. However, none of them appears inside the shell, and moreover, they are early B type stars with much weaker winds, thus their influence on the formation of the ring nebula through UV photon flux or stellar winds is negligible. Therefore, only LSS 1887 and WR 23 appear to be responsible for the formation of the bubbles.

Smith et al. (2000) suggested that the ionizing radiation and stellar winds from the massive stars near $\eta$ Car in the center of the Carina Nebula, have played a role in shaping the ISM up to distances as far as WR 23. However, the striking morphology of Anon(WR 23) and the location of the WR star within the nebula indicate that WR 23 has had a major contribution to the formation of its own ring nebula.

Finally, for the stellar wind parameters adopted for WR 23 and the ambient density listed in Table 2, a standoff distance of 2.2 pc can be derived for the bow shock (Wilkin 1996). We note that this value is smaller than the observed distance $(6.5 \mathrm{pc})$. Larger stellar wind parameters and/or smaller stellar velocities are needed to explain the observed standoff distance. A similar conclusion can be obtained for the case of LSS 1887 and RCW 52.

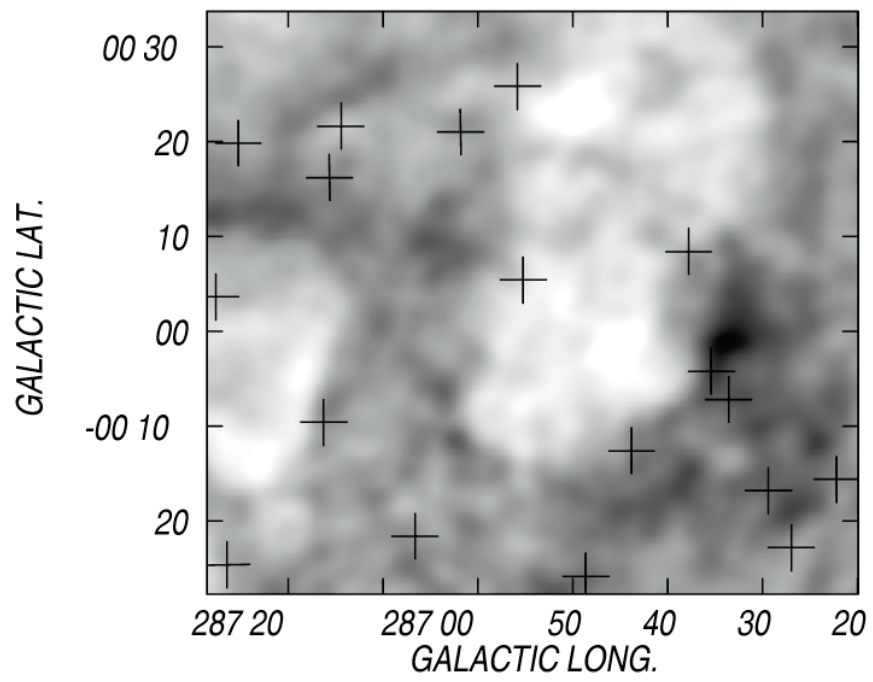

Fig. 6. Positions of the IRAS protostellar candidates, indicated by plus symbols, projected onto the HI column density distribution image in the region of the HI bubbles surrounding WR 23 and LSS 1887. The grayscale corresponds to the HI interval $(11.0-21.0) \times 10^{20} \mathrm{~cm}^{-2}$.

\subsection{Star formation}

Strong stellar winds may also induce star formation in their surroundings. Since few studies deal with star forming processes going on in the environs of optical ring nebulae, it is important to analyze the correlation of observed neutral gas structures with star formation indicators. Protostellar candidates can be identified as sources in the IRAS Point Source Catalogue which fit the criteria listed by, e.g., Junkes et al. (1992).

The positions of these protostellar sources are shown in Fig. 6 (plus symbols), superimposed onto the image showing the HI voids and shells related to Anon(WR 23) and RCW 52. Five IR sources are projected onto the HI shell associated with Anon(WR 23) (10379-5828, 10382-5827, 10893-5817, 10386-5838 and 10410-5828), while three are close to the inner border of the HI structure related to RCW 52) (10427-5817, 10441-5823 and 10439-5828). We note, however, that the distances of the protostellar candidates are unknown.

The IRAS protostellar candidates close to $286^{\circ} 35^{\prime},-0^{\circ} 5^{\prime}$ and RCW 52 coincide with other signspots of current active star formation listed by Avedisova (2002). The location of the protostellar candidates in the periphery of Anon(WR 23) behind the ionization front suggests that a stellar formation mechanism like the one first described by Elmegreen \& Lada (1977) may be taking place in the neutral shell.

\section{Conclusions}

We have detected the neutral gas counterparts of the optical ring nebulae Anon(WR 23) and RCW 52 blown by the strong stellar winds of the massive stars HD 92809 (WC6) and LS 1887 (O8V), respectively. The HI bubble linked to WR 23 is $13.3 \mathrm{pc}$ in radius and expands at $10 \mathrm{~km} \mathrm{~s}^{-1}$, while for the HI bubble associated with LSS 1887, the radius and expansion velocity are $6.3 \mathrm{pc}$ and $7 \mathrm{~km} \mathrm{~s}^{-1}$. In both cases, the HI bubbles 
appear external to the optical ionized gas ring nebulae. These new HI bubbles have similar parameters to those found around other WR and O-type stars (cf. Cappa et al. 2002).

A comparison between the values of the kinetic energy of the bubbles and the energy of the stellar winds $\left(E_{\mathrm{W}}\right)$ in Table 2 shows that, in both cases, the stellar wind power is enough to create the observed bubbles. The dynamical age derived for the neutral gas bubble surrounding HD 92809 suggests that the bubble was blown during the WR stage of the massive central star. We note that for most of the HI bubbles observed around WR stars, dynamical ages appear an order of magnitude larger than the duration of the WR phase of a massive star, suggesting that both the WR star and its massive progenitor have contributed to creating the bubbles (Cappa et al. 2003).

The large tangential velocities of WR 23 and LSS 1887, pointing towards the brighter borders of the optical nebulae, suggest that at least some regions of the nebulae are bow shocks created by the stellar motion.

Acknowledgements. We acknowledge the anomymous referee for very helpful comments. We thank T. Dame for making his CO data available to us. This project was partially financed by the Consejo Nacional de Investigaciones Científicas y Técnicas (CONICET) of Argentina under project PIP 607/98 and FCAG, UNLP under projects 11/G049 and 11/G072. The Digitized Sky Survey (DSS) was produced at the Space Telescope Science Institute under US Government grant NAGW-2166.

\section{References}

Avedisova, V. S. 2002, Astron. Zh, 79, 216

Brand, J., \& Blitz, 1993, A\&A, 275, 67

Cappa, C., Arnal, E. M., Cichowolski, S., Goss, W. M., \& Pineault, S. 2003, in A Massive Star Odyssey, from Main Sequence to Supernova, ed. van der Hucht, K. A., \& Esteban, C., IAU Symp., 212, 596

Cappa, C. E., Goss, W. M., Niemela, V. S., \& Ostrov, P. 1999, AJ, 118, 948

Cappa, C. E., \& Herbstmeier, U. 2000, AJ, 120, 1963

Castor, J. I. 1993, in Massive stars: their Lives in the Interstellar Medium, ed. J. P. Cassinelli \& E. B.Churchwell, ASP Conf. Ser., 35,297

Caswell, J. L., \& Haynes, R. F. 1987, A\&A, 171, 261
Condon, J. J., Griffith, M. R., \& Wright, A. E. 1993, AJ, 106, 1095

Crampton, D. 1972, MNRAS, 158, 85

Chu, Y.-H. 1982, ApJ, 254, 578

Chu, Y.-H., Guerrero, M. A., \& Gruendl, R. A. 2004, How does the Galaxy work? A Galactic Tertulia with Don Cox and Ron Reynolds, ed. Alfaro, Perez, \& Franco, Proc. workshop, in press

Dame, T. M., Hartmann, D., \& Thaddeus, P. 2001, ApJ, 547, 792

Draine B. T., \& Lee H. M., 1984, ApJ, 285, 89

Dyson, J. E. 1989, in Proc. Structure and Dynamics of the Interstellar Medium, ed. Tenorio-Tagle, G., Moles, M., \& Melnick, J. (Springer Verlag), IAU Coll., 120, 137

Eenens, P. R. J., \& Williams, P. M. 1994, MNRAS, 269, 1082

Elmegreen, B. G., \& Lada, C. J. 1977, ApJ, 214, 725

Feinstein, A., Marraco, H., \& Muzzio, J. C. 1973, A\&AS, 12, 33

García-Segura, G., \& Mac Low, M.-M. 1995, ApJ, 455, 145

Georgelin, Y. M., Lortet, M. C., \& Testor, G. 1986, A\&A, 162, 265

Goss, W. M., \& Lozinskaya, T. A. 1995, ApJ, 439, 637

Grabelsky, D. A. 1985, Ph.D. Thesis, Columbia University

Grabelsky, D. A., Cohen, R. S., Bronfman, L., \& Thaddeus, P. 1988, A\&A, 331, 181

Haynes, R. F., Caswell, J. L., Simons, L. W. J. 1979, Austr. J. Phys. Suppl., 45, 1

Hog, E., Fabricius, C., Makarov, V. V., et al. 2000, A\&A, 355, L27

van der Hucht, K. A. 2001, New Astron. Rev., 45, 135

Junkes, N., Fürst, E., \& Reich, W. 1992, A\&A, 261, 289

Lamers, H. J. G. L. M., \& Leitherer, C. 1993, ApJ, 412, 771

Lortet, M. C., Niemela, V. S., \& Tarsia, R. 1980, A\&A, 90, 210

Lundström, I., \& Stenholm, B. 1984, A\&AS, 58, 163

Marston, A. P. 1996, AJ, 112, 2828

Marston, A. P., Chu, Y.-H., \& García-Segura, G. 1994, ApJS, 93, 229

Massey, P., Johnson, J. 1993, AJ, 105, 980

Mathis, J. S., Cassinelli, J. P., van der Hucht, K. A., et al. 1992, ApJ, 384, 197

McClure-Griffiths, N. M. 2001, Ph.D. Thesis, University of Minnesota

McCray, R. 1983, Highlights of Astron., 5, 567

Mezger P. D., \& Henderson A. P., 1967, ApJ, 147, 471

Nugis, T., \& Lamers, H. J. G. L. M. 2000, A\&A, 360, 227

Prinja, R. K., Barlow, M. J., \& Howarth, I. D. 1990, ApJ, 361, 607

Rogers, A. W., Campbell, C. T., \& Whiteoak, J. B. 1960, MNRAS, 121,103

Smith, N., Egan, M. P., Carey, S., et al. 2000, ApJ, 532, L145

Vijapurkar, J., \& Drilling, J. 1993, ApJS, 89, 293

Walborn, N. 1995, in The $\eta$ Car region: a laboratory of Stellar Evolution, ed. Niemela, V., Morrell, N., \& Feinstein, A., Rev. Mex. Astron. Astrofis., Conf. Ser., 2, 51

Wilkin, F. P. 1996, ApJ, 459, L31 